\title{
LECTINS: A REVIEW ON ITS PHYSICO-CHEMICAL PROPERTIES
}

\section{SINGH G AND SINGH RD*}

1: School of Bioengineering and Biosciences, Lovely professional university, Phagwara,

$$
\text { Punjab (India) }
$$

*Corresponding Author: Rattan Deep Singh: E Mail: rattan.19383@lpu.co.in

Received 11 ${ }^{\text {th }}$ March 2021; Revised 10 ${ }^{\text {th }}$ April 2021; Accepted 10 ${ }^{\text {th }}$ May 2021; Available online $1^{\text {st }}$ Jan. $_{2022}$

\section{https://doi.org/10.31032/IJBPAS/2022/11.1.5833}

\begin{abstract}
Lectins are the protein compounds with carbohydrate binding unit and shows high degree of specificity towards sugar moieties. The word lectin is come from Latin word legree which means to select. Lectins are made up of four similar subunit hence called tetramer compound. Lectins can be isolated from plant, microbes and animal and have immense role in the field of microbiology and cancer biology. Lectins may be used for the detection and study of glycosylated blood group antigens. Several sugars molecules like fructose, mannose, sialic acid, N-acetyl glucosamine, and $\mathrm{N}$-acetylgalactosamine and glycan complex binds with lectins as per their specificity .Concanavalin A (Con-A) is a hemagglutinin that was first purified from jack bean. Lectins are classified according to a specific group of monosaccharide therefore which is a best hapten inhibitor.Lectins are resistant to gastric acid and proteolytic enzymes. This review comprehensive account of lectins classification /distribution, hemagglutation activity, sugar specificity, Antinutritional property and its application in the field of medicinal biology.
\end{abstract}

Keywords: Lectins, Haemagglutination, Metalloproteins, Lectinology, Glycoprotein, Mitogenic

\section{INTRODUCTION}

Lectins are defined as non-catalytic member of a protein family that are ubiquitously in nature, has a capacity to bind specific carbohydrate moiety therefore combining with glycol component on the cell surface without changing their covalent structure [1]. Lectin comes from Latin word legere which to select Lectins was 
found to be polyvalent or univalent. Their origin is non-immunogenic, but they interact with different other proteins, carbohydrates and saccharide molecules and are widely known for their agglutination activities. Lectins are known to have cell agglutination and precipitation properties as a result of which lectin binds with various molecules of sugars of glycoproteins as a result of it each molecules of lectins has carbohydrate binding sites which form a cross linking connection between the cells and sugar containing macromolecules [2]. Lectins generally show antifungal, mitogenic, immunomodulatory, and antitumor properties, anti-HIV-1 reverse transcriptase and antineoplastic activities. On the basis of carbohydrate binding domain lectins are classify into four types as merolectin, hololectin, chimerolectin, and superlectin out of which chimerolectin is most abundantly found in plants [3].

Mostly lectins are isolated from seed cotyledons, where they are found in the cytoplasm or they are present in protein bodies, and play an important role to reserve proteins in the regulation of plant development. Some lectins are capable to induce mitosis in the cell.Lectins may be used for the detection and study of glycosylated blood group antigens. Plants are rich in lectins and are therefore referred to as phyto-hemagglutinin due to their ability to agglutinate erythrocytes in a carbohydrate manner. Interaction involve for the recognition of carbohydrate domain includes van der waals forces and the formation of hydrogen bonds at the active site of lectin between the sugar hydroxyl terminal and the amino acid residue. If erythrocytes are involved, the phenomenon is referred to as haemagglutinin. Ingestion of lectins by animals can degenerate the cell membrane with anti-nutritional consequences which can contribute to significant physiological results. They act as defence proteins and show toxicity against various predators. This class of protein is found in both flora and fauna as well as in bacteria and fungi and are cosmopolitan with in plant kingdom $[4,5]$.

Plant lectins act as the method to differentiate between malignant and benign tumors, and to assess the metastasis by glycolytic stage. Certain plant lectins are used to prevent fungal growth directly. Some plant lectins are heat resistant, heat processing can reduce their toxicity [1]. Term lectins was coined by Boyd and shapleign in 1954 to show that a class of protein which is non immunoglobulin in nature will bind with carbohydrate without its modification [6]. Lectins are isolated from various parts of the plants like leaves, bark, roots, seeds and flowers. Roots, bulbs, tubers, barks and leaves of Sambucus, Solanum, Allium, Cladrastis, 
Viscum album, respectively are studied and known to provide a rich lectin source. Lectins derived from plant are categories as a group of heterogeneous molecules which shows different biochemical properties and carbohydrate specificity. Mostly are rich in leguminous family and are composed of monomer in a form of dimer or tetramer of molecular weight $25 \mathrm{KDa}$ [7]. Inflammatory glioma cell toxicity asorelaxant activity is shown in diocleniae which is further categories as a class of lectin and shows glu/mannose affinity [8, 9].

Lectins has a wide variety of application therefore can protects plants against attack of pests and other diseases through a specific protein carbohydrate interaction mediators. The mechanism of recovery involves a specific interaction between protein receptors and saccharide residues. Through the cell wall lectins can bind a cascade of biological reactions to accessible carbohydrate residue.

\section{DISTRIBUTION}

Lectins are widely distributed in the plant kingdom and most widely studied in Leguminoceae family. This class of proteins has been isolated from many plants and animals [9]. Lectins are highly specific to other sugar moieties. Biological properties of lectins are determined by the ability to interact with carbohydrates while combining with glycol components on the cell surface. Lectins are highly specific to other sugar moieties. Biological properties of lectins are determined by the ability to interact with carbohydrates while combining with glycol components on the cell surface. Lectins are specific to monosaccharides or oligosaccharides [10] Lectins are present in various plant tissues with different biological properties. The major source of lectins is seeds and legumes, but lectins from plants have been purified from different parts: barks, leaves, fruits, tubers, roots, rhizomes, cotyledons and phloem exudate. Plant lectins isolated from algae, lichens and ferns have distinctive properties depending upon their binding affinity with a particular saccharide.They recognize the cellular and molecular levels within the biological systems and play numerous roles in activities that involve cells, carbohydrates, and proteins and work as a mediator for fungi, bacteria and viruses to attach and bind to their targets [3].

The agglutination and precipitation characteristics of lectins are similar to those of antibodies, which thus specifically inhibited by lower molecular weight compounds called haptens [10]. Initially, lectins were isolated using organic solvents, salts and acids, but with the progressive advancement in the area of analytical chemistry, preparative chromatographic methods like, ion exchange, gel filtration 
(size exclusion chromatography) and affinity adsorbents are now being used for better and substantial isolation and purification of lectins. Common methods employed for isolating enzymes by means of enzyme immobilization could also be used to isolate lectins. Therefore crosslinked dextran's found potential agents for purification of Glc-binding plant lectins [11].

Lectins are distributed in the carbohydrate specific groups of fucose, mannose, sialic acid, N-acetyl glucosamine, and Nacetylgalactosamine and glycan complex.

Table 1: Showing the classification of lectins based upon their specific binding sites with respect to their carbohydrate binding affinity and their structures

\begin{tabular}{|c|c|c|c|}
\hline S. No. & $\begin{array}{c}\text { Number of carbohydrate } \\
\text { binding sites }\end{array}$ & $\begin{array}{c}\text { Binding affinity to } \\
\text { carbohydrate moiety }\end{array}$ & Structure \\
\hline 1. & Hololectins & Glucose & Amaranthine \\
\hline 2. & Merolectins & Galactose & Chitin binding lectin \\
\hline 3. & Chimerolectins & L-fucose & $\begin{array}{c}\text { Monocot mannose } \\
\text { binding lectin }\end{array}$ \\
\hline 4. & Super-lectins & Sialic acid & $\begin{array}{c}\text { Type-2 ribose inactivating } \\
\text { lectin }\end{array}$ \\
\hline
\end{tabular}

In case of lectins isolated from plants may consist of two classes

- Freely soluble to membrane and are similar with seed lectins.

- Intercalated into bilayer of membrane which required detergent for solubilizing it and are quite different from seed lectins.

Roots, bulbs, tubers, barks and leaves of Sambucus, Solanum, Allium, Cladrastis, Viscum album, respectively are studied and known to provide a rich lectin source. Heterologous systems show expression of plant recombinant lectins. Few lectin which are isolated from plant are known for their lethality, particularly ricin and abrin. .But most widely lectins are extracted from plants due its defence mechanism against seed predators and seedling development, and found in every part of plant i.e., leaves, tubers, root, stem etc. Amount of lectin is maximum when the seed is mature while minimum in the germination stage. Lectin which are isolated from seeds can bind to $\mathrm{N}$-acetyl galactosamine and which are isolated from vegetative parts bind to both $\mathrm{N}$-acetyl galactosamine and $\mathrm{N}$-acetyl glucosamine. Per molecules of lectin in the composition of molecular weight, structure, and number of sugar binding sites therefore refers to as protein [3]. Subunits of lectins differ greatly, in Limulus lectin have 18 similar subunits while Abrusprecatoriusagglutinin form differ in 2 S-S linked subunits, and only one of them can bind to sugar moiety. Some lectins are free from covalently bound sugar such as con A, WGA, PNA etc. and rich in aspartic acid, serine ,hydroxyl, proline, and also have functional group i.e., amino, carboxyl, 
hydroxyl or thiol group. Range of oligomerization and sugar specificity exhibit in legume lectins. Some lectins have higher tolerance of agglutination activity towards erythrocytes by treating with proteolytic enzymes such as trypsin. Therefore proteolytic treatment and transformation were suggested to lead to exposure or rearrangement of lectin receptor sites which are cryptic in normal cells. Receptor lectins have been shown to be clustered in transformed cells and randomly distributed in normal cells [12].

\section{Phyto-haemagglutination}

The $\mathrm{pH}$ as well as temperature stability of lectins can be assessed with haemagglutination assay which further determines the environment for application of lectins in biotechnology. Phytohaemagglutination is a common method to study lectin interaction with sugars. This method also reveals the decreased affinity of lectins towards RBC due to binding of specific sugars with lectins. Several other techniques are their which can find sugar specificity of lectins are based on immobilization of carbohydrates on a metal coated glass prism as done in surface Plasmon resonance method and employing enzymelinked adsorbent assay using sugar acrylamide conjugates.

Unlike growth factors, such as EGF, TGF, IGF that are targeting the tissues are diluted in the blood plasma and hence they are not carried to their specific site of action. In contrast, the proteins, such as lectins can be administered orally, are resistant to degradation by proteolytic enzymes in the gut and have promotive effects on the gut as the target organ [13].

Cereal and other legume lectins increases the interaction of the human gut to various bacterial antigens, peptide fragments derived from milk and cereal grain proteins. Several lectins are capable of activating T-cells in a Human Leukocytes antigens (HLA in humans nucleated cells represents the major histocompatibility complex class I molecules) [13]. Lectins may attach to certain fungal hyphae and prevent the use of nutrients and the increase fungal growth precursors.

Concanavalin A (Con-A) is a first hemagglutinin which was isolated from jack bean. Con-A was found to give a negative effect in a haemagglutination process, when it dissolved in a sucrose solution .Studies and experiments shown that the presences of carbohydrate on the erythrocytes surface is an important factor for agglutination [14].

Lectins which are isolated from Legume are generally metalloproteins. In which each subunit contains a $\mathrm{Mn} 2+$ and a $\mathrm{Ca} 2+$ ion, and are essential for the carbohydratebinding activity of lectins. There are many but not all legume lectins are $\mathrm{N}$ - 
glycosylated [15]. Eliminating lectins from the diet can reduce peripheral antigenic stimulus and symptoms of rheumatoid arthritis may be alleviated. Their exclusion from the diet can have beneficial health effects such as lowering the level of $\mathrm{C}$ reactive proteins and blood pressure. Sumner and Howell in 1936 isolated lectin from jack bean which was the first crystalline form of lectin, observed that when the glycogen reacted with protein showed difficulty in haemagglutination activity with carbohydrate in stroma protein. Genus Phaseolus species is the most important source of lectin which in its pure form can be used to stimulate cells to undergo mitotic division [16].

Lectins are extractable from plants but their $\mathrm{pH}$ must be maintained using buffers to stabilize the agglutination reactions. Their extraction time and the temperature depend on the solubility and their stability. Above 27C their agglutination activity may get disturbed. The reversed micelle system of anionic sodium di (2-ethylhexyl) sulfosuccinate surfactant in isooctane may also be used to extract lectins, in which protein solubilisation is highly dependent on $\mathrm{pH}$, surfactant concentration and micelle size [17].

Partially purified phyto-haemagglutinin inhibits the development of larvae of Callosobruchus maculatus [12]. Lectins have greater affinity towards its receptor because of its small change in the receptor of carbohydrate structure. Lectins in the form of pro-lectins are synthesized in the ER where the 20-30 amino acid ladder sequence is removed. Lectin bind to cell surface and form several bridge between applied cells, resulting in the agglutination that can detect the interaction of lectin with cells more easily. Further in Golgi apparatus post translational modification of carbohydrates take place. In the assay mixture, metal ions are also used as well because some lectins have shown that these ions require for their activity [15]. Some lectin binding site with D-pyranose oligomer may be inhibited more efficiently than monomer suggesting that the lectin detect a sequence of sugar residue instead of monosaccharide.

\section{Sugar specificity}

The specificity of lectins is typically expressed by its simple monosaccharide subunits which helps in inhibiting its effect. Actual structure of lectins are recognized by its binding sites, once it combines with its natural ligand produces a more complex structure than simple monosaccharide. Lectins which are similar in their sugar specificity towards the monosaccharide may have different affinity toward particular disaccharide and glycopeptide. (V.villosa lectins first bind to glycoprotein then to oligosaccharide). Hence appears to recognize its carbohydrate subunit with the 
amino acid to which they are linked latter on. Oligosaccharides are having characteristic property of rotation around glyosidic bond and can generate by binding with lectins.

ConA and lentils lectin are similar in their sugar specificity but lectins isolated from conA binds to glycoprotein whereas lentils binds to glycoprotein and glycophorin Lectin can also bind to non-carbohydrate ligand. Lectins can react preferentially with non-reducing sugar and also with internal component of carbohydrate chain [18]. Lectins also show anomeric specificity therefore react with alpha or beta anomers of their respective monosaccharide. Some lectins show metaloprotein activity therefore required metal ion $\mathrm{Ca}^{+} \mathrm{Mg}^{+}$(pea lectins and Con A). Due to differences in their structure and chemical properties lectins also differ in their biological and physicochemical property. Lectins are classified according to a specific group of monosaccharide therefore which is a best hapten inhibitor. Lectin can lead to cell lysis when the specific carbohydrate chains are on the erythrocyte surface. Due to absences of complementary carbohydrate residue in human blood cells, lectins have soluble factors that can agglutinate R.B.C. and even yeasts [19]. Some lectins show hemolytic activity which are galactose specific [20].
Lectins are generally classified into five specificity groups, according to the monosaccharide subunit for which they assets the highest affinity: mannose, galactose/N-acetylgalactosamine, $\mathrm{N}$-acetyl glucosamine, fucose, and Nacetylneuraminic acid (all sugars excluding fucose are in D-configuration, fucose is in L-configuration). These six are constituents of the glycan's present on the surfaces of every eukaryotic cell. Lectins specific for mannose with 2-hydroxyl configuration bind with glucose but the ones that bind with galactose are of 4hydroxy configuration. Groups with the same specificity of lectins show different affinity towards oligosaccharides, few oligosaccharide derivatives were identified from above monosaccharides. Specificity for other sugars has been rarely encountered. The affinity of lectins to monosaccharides is usually weak, with association constants in the mill molar range, whereas that to oligosaccharides is often much higher, up to three orders of magnitude. The mannose specific lectins constitute a growing group of proteins from Monocotyledonous plants, whose interaction is especially towards mannose, a fact not observed in glucose/mannose lectins [21]. In addition, lectins have been demonstrated to interact with sialic acid and some derivatives, although glucose and 
galactose specific lectins also show ability to interact with this acid [22].

\section{Anti-nutritional property}

Lectins are extremely anti-nutritional substances that effect various herbivorous organisms. Its property is associated with its high chemical resistance action in monogastric, ruminants and insects digestive tubes. It has been proven that lectin isolated from plants act as biological agent against insect attack. As transgenic plants which are insect resistant are produced by the expression of lectin genes which results in increase crop production and decreased the use of pesticides. Although few are selected on the basis of high resistance to disease or predators in plants, simultaneously having low toxicity to higher mammals.

As partially or uncooked kidney beans causes food poisoning which shows adverse effect on gastroenteritis and is named as PHA by loss of epithelial resistance and becomes fatal when ingested in higher concentration. To decrease the amount of PHA before consumption heating is used which is a traditional method but it is relatively resistant to thermal denaturation. Lectin activity cannot be abolished by slow heating or by heating $5 \mathrm{~h}$ at $91^{\circ} \mathrm{C}$; whereas by heating at $100^{\circ} \mathrm{C}$ for $5 \mathrm{~min}$ or by ultra-high pressureits activity can be fully abolished [23]. High amount of PHA intake can cause harm to the intestinal wall which may result in exponential growth of coliform bacteria in lumen and internal organ also. After addition of lectins in diet may affect the immune system due response generated by a strong humoral anti lectin IgG-antibody.

\section{Applications}

Lectins play a major role in recognizing the cellular and molecular levels within the biological systems and in the activities that involve cells, carbohydrates, and proteins to work as a mediator for fungi, bacteria and viruses to attach and bind to their targets. The main role lectin is to bind with glycan component for signaling, defense and stress response. They are reported to have a high degree of haemagglutination and antimicrobial effect by binding to the cell surface. They have ubiquitous nature and are abundant in many plants.

Several studies have reported that Plant lectins are resistant to proteolytic degradation in the gut and therefore can remain active throughout their passage in the gastrointestinal tract [24]. They interact on the surface of gut epithelial cells with the highly glycosylated receptors which result in compartmentalization of the gastrointestinal tract, and can affect the different parts of the gut. Glycosylation of gut surface cells through plant lectins could help in determining molecular and cellular mechanism of lectin interaction with the gut epithelium. 
Lectin isolated from plant tends to prevent viruses in human and animal. Lectin isolated from Boodleacoacta (BCA), has high specificity to inhibit HIV-1 (Human Immunodeficiency Virus) and showed a strong anti-influenza activity by directly binding to the viral envelope which is highly specific in mannose type $\mathrm{N}$-glycan [25].

Lectins has variety of application and are capable of recognizing other molecules applicable for research ,in structural analysis, in vivo as well as in vitro analysis in different fields such as molecular and cell biology, biotechnological, immunology, pharmacology, medicine, clinical analysis, nanotechnology as well as in systems for drug release[26].

Lectins have several applications in biological sciences, both as inter-mediators or as agents. Numerous reports are being available suggesting lectins as prominent anti-insect and anticancer agents [27].

Due to their high end specificity with carbohydrate residues regardless of presence in solutions or on cellular surfaces, lectins could be employed as useful tools in bioscience and biomedicines. Genetic engineering techniques have been used to transfer insecticidal lectin transgenes and produce genetically engineered crop plants that are resistant to pathogenic response. As per current reports, only a few lectins like Con-
A and PHA are potentially used to initiate specific cellular responses.

A major use of virulent lectins such as ricin used for the resistant's of animal cell lines selection and accessing modified glycan related structure glycoprotein with novel glycosyltransferases. They are useful tool to detect glycosylation mechanism and to generate therapeutic glycoproteins with desired carbohydrate structures.

\section{Lymphocyte activation}

Lymphocyte activation is done by inducing the synthesis of specific proteins such as enzymes, cytokines or interleukins are well known applications. Lectins isolated from tulip bulbs and autumnal tubers have interesting mitogenic characteristics, but are not commonly used. While lectin isolated from Calistoga Sepium has a strong mitogenic activity but less cytotoxicity compared to CA and PHA and therefore it is used in far wider concentration. Some lectins do not only affect cells but also have specific effects on entire organisms when they are injected or ingested orally [27].

2. Detection and isolation of glycoconjugates

Lectins can be used to identify and isolate glycol-conjugates which are complementary to lectin binding sites with carbohydrate structure. Detection of carbohydrate is based on the binding capability of lectins with carbohydrates 
present on surfaces or in the form of glycolconjugate followed by visualization of bound lectin which may be direct or indirect. Histopathology techniques employs the detection of carbohydrates based on appearance of glycan residues present on transformed cell surface. Therefore glycol-conjugates and glycopeptides could be fractionated and purified by lectins [27].

\section{Tumor targeting tools}

.Early detection for tumor metastasis is extremely vital for the survival of cancer patient. In most of cases the metastases don't seems to be detectable till they need to reach a definite size ${ }^{25}$. Cellular glycosylation alterations can be studied by the use of lectins along with monoclonal antibodies. They can easily and firmly detect the different chains of sugars and hence are more valuable than the monoclonal antibodies. HPA binding can be studied in breast cancer, lung cancer and colon carcinoma. Besides HPA many different lectins are capable of detecting tumors and cancerous cells. Among different type of oligosaccharide, fucosylation is shown to be one of the most important type of carcinogenesis and cancer metastasis, and derivative fucosylated haptoglobin is known as a marker for carcinoma [27].

Immobilized lectins in affinity chromatography
The interaction between lectins and carbohydrates involves non-covalent hydrogen bonding, as well as hydrophobic, electrostatic, and van der Waals interactions this does not allow modification of ligands during purification process. Con A-Sepharose and Lentil Lectin Sepharose 4B column are generally used for lectin purification and characterisation. Several studies has reported that lectins can be separated on the basis of affinity chromatography as lectins are specific for particular sugar molecules [27].

\section{Histochemistry and Cytochemistry}

Lectins can be labelled with a fluorescent dye, such as fluorescein iso thiocyanate (FITC), and viewed directly by using a fluorescence microscope, or they are conjugated to enzymes (such as horseradish peroxidase) or haptens (such as biotin and digoxigenin), followed by second incubation with proper substrates to become detectable by light microscopy or electron microscopy with peroxidase, ferritin, or colloidal gold. Lectins conjugated to digoxigenin could be detected by antidigoxigenin antibodies that may be complexed to particles of colloidal gold for electron microscopy [27].

\section{Lectin Blotting}

The procedures are similar to those of immunoblotting with only a few discrepancies. After electrophoresis, 
proteins were transferred to nitrocellulose membranes, equilibrated and incubated with biotinylated lectins, followed by washing and incubation with streptavidinalkaline phosphatase conjugate. In this process, biotinylated lectins are analogous to the first antibodies in immunoblotting, but biotinylated lectins are much more easily prepared and more economically available. Immunoblotting covers a wider range of proteins, while lectin blotting is more specific to glycoproteins. With this technology, lectins can help identify certain glycan's in certain pathways or organelles semi quantitatively [27].

\section{Lectin-Based Biosensors}

Biological interactions between lectins and glycan's are transformed into a signal that could be easily quantitated, analysed, and presented in a user-friendly way. Fluorescent Con A was obtained by attaching a photo affinity labelling reagent to the sugar-binding pocket of Con A followed by UV irradiation and subsequent treatment with dithiothreitol (DTT) to yield a mercaptobenzyl site which is modified with an iodoacetylated dansyl group. The disadvantage of this method is that labelling of lectin is required, which may increase unwanted variability [27].

\section{Lectin and Insects}

Lectin can be used as insecticidal molecules against various species and used in the production of transgenic plant through $\mathrm{N}$-acetyl-D-glucosamine binding. It has been reported that lectin isolated from mushroom have greater affinity towards D- galactose and lactose and to lesser N-acetyl-D-glucosamine, result adverse effect on Drosophila melanogaster and pea aphid Acyrthosiphon pisum [28].

\section{CONCLUSION}

Lectins can be a useful tool and add up as an advanced method for many biological and therapeutic uses. Commercially available agarose containing natural polysaccharide Gal is used as an affinity adsorbent for Gal-binding lectins.

Lectin can be isolated from plant leaves due to the identical $\mathrm{N}$-termini of cross reactive material subunit that closely resembles the same binding specificity and has a higher isoelectric point than that of seed lectin.

Lectin can be used for the treatment of cancer, $\mathrm{ABO}$ blood typing, and microarrays. Lectin plays a major role in inhibiting the adherence and invasion of bacteria therefore recognition of membrane to glycoconjugate of microorganisms which allows lectin to act as an inhibitory age.

\section{REFERENCES}

[1] Mazalovska M, Kouokam JC. Plantderived lectins as potential cancer therapeutics and diagnostic tools. BioMed Research International. 2020 May 15; 2020. 
[2] Adenike K, Eretan OB. Purification and partial characterization of a lectin from the fresh leaves of Kalanchoecrenata (Andr.) Haw. Journal of Biochemistry and Molecular Biology. 2004 Mar 31; 37(2): 229-33.

[3] Gautam AK, Shrivastava N, Sharma B, Bhagyawant SS. Current scenario of legume lectins and their practical applications. Journal of Crop Science and Biotechnology. 2018 Sep; 21(3): 217-27.

[4] Tsaneva M, Van Damme EJ. 130 years of Plant Lectin Research. Glycoconjugate Journal. 2020 Aug 29: 1-9.

[5] Singh RS, Walia AK. Microbial lectins and their prospective mitogenic potential. Critical reviews in microbiology. 2014 Nov 1; 40(4): $329-47$.

[6] Boyd WC, Shapleigh E. Specific precipitating activity of plant agglutinins (lectins). Science. 1954 Mar 26; 119(3091):419.Sharon N, Lis H. Lectins: cell-agglutinating and sugar-specific proteins. Science. 1972 Sep 15; 177(4053): 949-59.

[7] Sharon N, Lis H. Carbohydrates in cell recognition. Scientific American. 1993 Jan 1; 268(1): 82-9.

[8] Osterne VJ, Silva-Filho JC, Santiago MQ, Pinto-Junior VR, Almeida AC,
Barreto AA, Wolin IA, Nascimento AP, Amorim RM, Rocha BA, Delatorre P. Structural characterization of a lectin from Canavalia virosa seeds with inflammatory and cytotoxic activities. International journal of biological macromolecules. 2017 Jan 1; 94: 271-82.

[9] Pinto-Junior VR, Osterne VJ, Santiago MQ, Correia JL, PereiraJunior FN, Leal RB, Pereira MG, Chicas LS, Nagano CS, Rocha BA, Silva-Filho JC. Structural studies of a vasorelaxant lectin from Dioclea reflexa Hook seeds: Crystal structure, molecular docking and dynamics. International journal of biological macromolecules. 2017 May 1; 98: 12-23.

[10]Cummings, R.D., Darvill, A.G., Etzler, M.E. and Hahn, M.G., 2017. Glycan-recognizing probes as tools.

[11] Rüdiger H, Gabius HJ. Plant lectins: occurrence, biochemistry, functions and applications. Glycoconjugate journal. 2001 Aug; 18(8): 589-613.

[12]Reisner Y, Lis H, Sharon N. On the importance of the binding of lectins to cell surface receptors at low lectin concentrations. Experimental cell research. 1976 Feb 1; 97(2): 445-8.

[13] Mbae KM, Umesha S, Manukumar HM. Therapeutic properties of 
lectins in herbal supplements.

Phytochemistry Reviews. 2018 Jun

$1 ; 17(3): 627-43$

[14]Peumans WJ, Stinissen HM. Gramineae lectins: occurrence, molecular biology and physiological function. Progress in clinical and biological research. 1983; 138: 99.

[15] KF CC, Ransom RC, Longaker MT. Lectins bring benefits to bones. eLife. 2016; 5.

[16] Sharon N. Glycoproteins. Scientific American. 1974 May 1; 230(5): 7887.

[17] Kenmochi, E., Kabir, S. R., Ogawa, T., Naude, R., Tateno, H., Hirabayashi, J., and Muramoto, K. (2015). Isolation and biochemical characterization of Apios tuber lectin. Molecules, 20, 987-1002.

[18]Goldstein IJ, So LL. Proteincarbohydrate interaction: III. Agar gel-diffusion studies on the interaction of concanavalin $\mathrm{A}$, a lectin isolated from jack bean, with polysaccharides. Archives of Biochemistry and Biophysics. 1965 Aug 1; 111(2): 407-14.

[19] Brown, J.C. and Hunt, R.C. (1978). Lectins. Int Rev Cytol., 52: $277-$ 337.

[20] Hatakeyama T, Sato T, Taira E, Kuwahara H, Niidome T, Aoyagi H. Characterization of the interaction of hemolytic lectin CEL-III from the marine invertebrate, Cucumaria echinata, with artificial lipid membranes: involvement of neutral sphingoglycolipids in the poreforming process. The Journal of Biochemistry. 1999 Feb 1; 125(2): 277-84.

[21]Chandra NR, Ramachandraiah G, Bachhawat K, Dam TK, Surolia A, Vijayan M. Crystal structure of a dimeric mannose-specific agglutinin from garlic: quaternary association and carbohydrate specificity. Journal of molecular biology. 1999 Jan 22; 285(3): 1157-68.

[22] Konami Y, Yamamoto K, Osawa T, Irimura T. Strong affinity of Maackia amurensis hemagglutinin (MAH) for sialic acid-containing Ser/Thr-linked carbohydrate chains of N-terminal octapeptides from human glycophorin A. FEBS letters. 1994 Apr 11; 342(3): 334-8.

[23] Van Buul VJ, Brouns FJ. Health effects of wheat lectins: A review. Journal of Cereal Science. 2014 Mar 1; 59(2): 112-7.

[24] Gabius HJ. Lectins: a primer for histochemists and cell biologists. Histochem Cell Biol. doi: http://dx. doi. org/10.1007/s00418-016-15246. 2017. 
[25] Mitchell CA, Ramessar K, O'Keefe BR. Antiviral lectins: Selective inhibitors of viral entry. Antiviral research. 2017 Jun 1; 142: 37-54.

[26] Shanmugavel S, Velayutham V, Kamalanathan T, Periasamy $M$, Munusamy A, Sundaram J. Isolation and analysis

of mannose/trehalose/maltose specific lectin from jack bean with antibruchid activity. International journal of biological macromolecules. 2016 Oct 1; 91: 14.

[27]Dan X, Liu W, Ng TB. Development and applications of lectins as biological tools in biomedical research. Medicinal research reviews. 2016 Mar; 36(2): 221-47.

[28] Santos AF, Da Silva MD, Napoleão TH, Paiva PM, Correia MD, Coelho LC. Lectins: Function, structure, biological properties and potential applications. 